\title{
Oxidation of Fe Whiskers and Surface Diffusion Observed by Environmental TEM
}

Aram Yoon ${ }^{1,2}$, Xiaofeng Zhang ${ }^{4}$, Wenpei Gao ${ }^{1,2}$, Jianbo Wu ${ }^{1,2,3}$, Yung-Tin Pan ${ }^{3}$, Hong Yang ${ }^{3}$ and Jian-Min Zuo ${ }^{1,2}$

${ }^{1}$ Dept of Materials Sci. and Eng., University of Illinois, Urbana-Champaign, IL 61801

${ }^{2}$ Materials Research Laboratory, University of Illinois, Urbana-Champaign, IL 61801

${ }^{3}$ Dept of Chemical and Biomolecular Eng., University of Illinois, Urbana-Champaign, IL 61801

${ }^{4}$ Hitachi High Technologies America Nanotechnology System Division, Pleasanton, CA 94588

Metal oxidation is one of the spontaneous chemical reaction, which is hard to control. Most metals inevitably have at least a few layers of oxides on the surface in natural environment. Because of the large impact of metal oxidation on infrastructure and transportation, surface oxidation and its role on structure and property have attracted significant attentions on the fundamental aspects of oxygen diffusion into the metal or metal ion transport $[1,3]$. Furthermore, iron/iron oxide is an attractive material for application, such as photo catalysis and electrode for lithium batteries [2], so the study of oxidation Fe material is also interesting for nanotechnology [3]. However understanding Fe oxidation in small length scale so far has been limited, since it is hard to observe ionic transport directly due to the spatial and temporal resolution limit.

Here, we present an in situ observation of iron oxide growth on an iron whisker. (Figure 1a) Experiment was carried using Hitachi H-9500 ETEM with a LaB6 emitter, operated at $300 \mathrm{kV}$. The special holder designed for the ETEM provide the gas injection and heating environment directly on the sample. We injected the air into the TEM to the pressure of $2 \times 10^{-3} \mathrm{~Pa}$, while heating the sample to $\sim 350{ }^{\circ} \mathrm{C}$. The images were recorded using the Orius SC200 camera from Gatan in Pleasanton, CA.

The Fe whisker had a thin crystalline surface layer initially. On top of this, a thin amorphous layer was observed on the iron whisker. When heated, oxide island started to popping up at seemingly random position sporadically, which is captured in Figure 2, Then, the island of the iron oxide rapidly grew and merged each other until it covered whole whisker to make fish-scale like shell. Also, we observed large volume expansion up to $150 \%$ as the thickness of the shell reached up to $50 \mathrm{~nm}$ (Figure 1b).

More interestingly, we have observed surface diffusion on the oxide islands and propagation of the lattice. We observed the growth that fits with the step growth model developed by Kossel, Stranski and Volmer (KSV model) (Figure 3). The propagation direction is usually from the whisker to the surface, but a few grew from outside (vacuum) to inside (whisker). In addition, some of the lattice step remained incomplete, leaving behind a stepped surface. The growth also slows down significantly after reaching some thickness before it stops. No further growth is seen even as we increase temperature or pressure. The preliminary results here suggest that iron diffusion is the key factor for the oxidation. Further, in-situ observation well enable us to determine the kinetic parameters and obtain a direct picture of the dynamics of the Fe oxidation [4].

\section{References:}

[1] G. Zhou et al., Chem. Commun. 49 (2013), 10862.

[2] M. H. Oh, et al., Science 340 (2013), 964.

[3] A.Pratt, et al., Nat. Mat. 13 (2014), 26. 
[4] The ETEM is supported by the NSF MRI Grant NSF DMR 12-29454. WPG is supported NSF DMR 0449790. JBW and JMZ are partially supported by DOE BES under contract DEFG02-01ER45923.
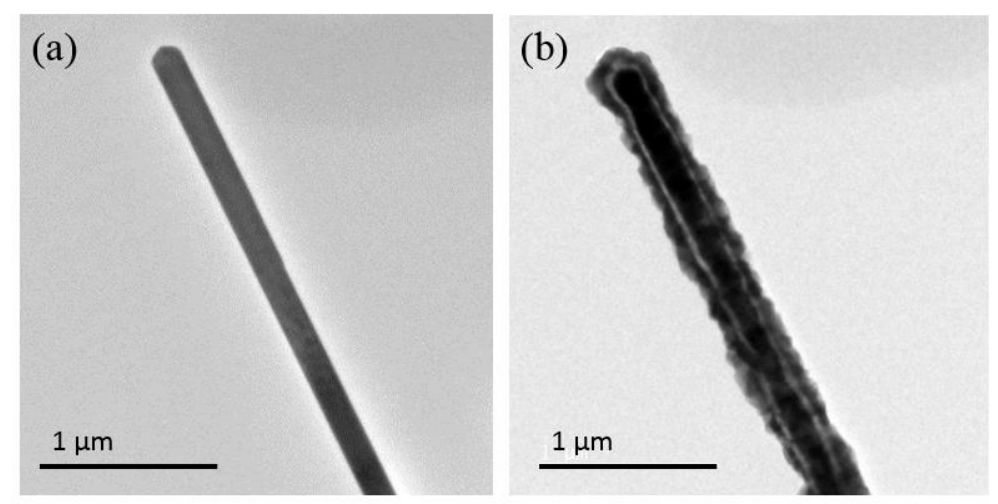

Figure 1. (a) Iron whisker before oxidation, (b) after completion of oxidation
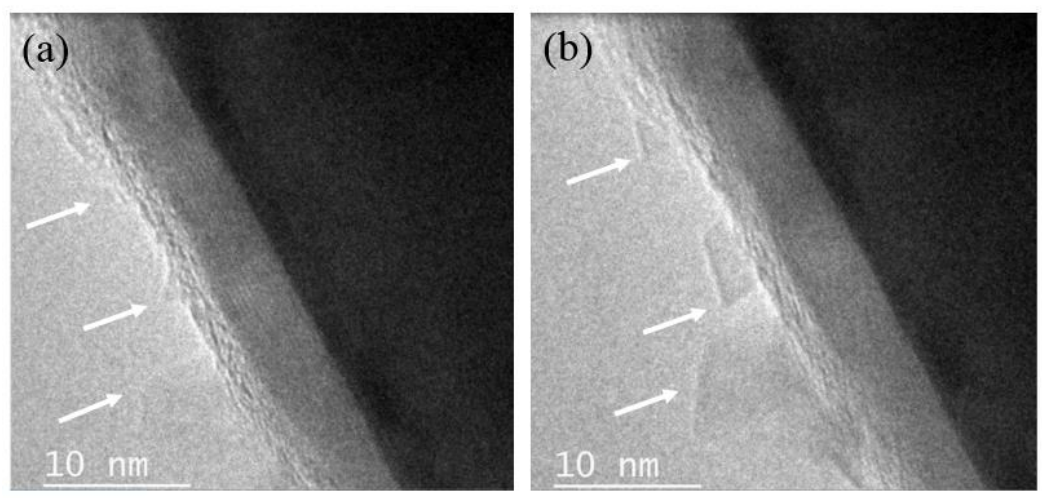

Figure 2. (a) Initial stage of the oxidation at 20 seconds after heating (b) 30 seconds after heating
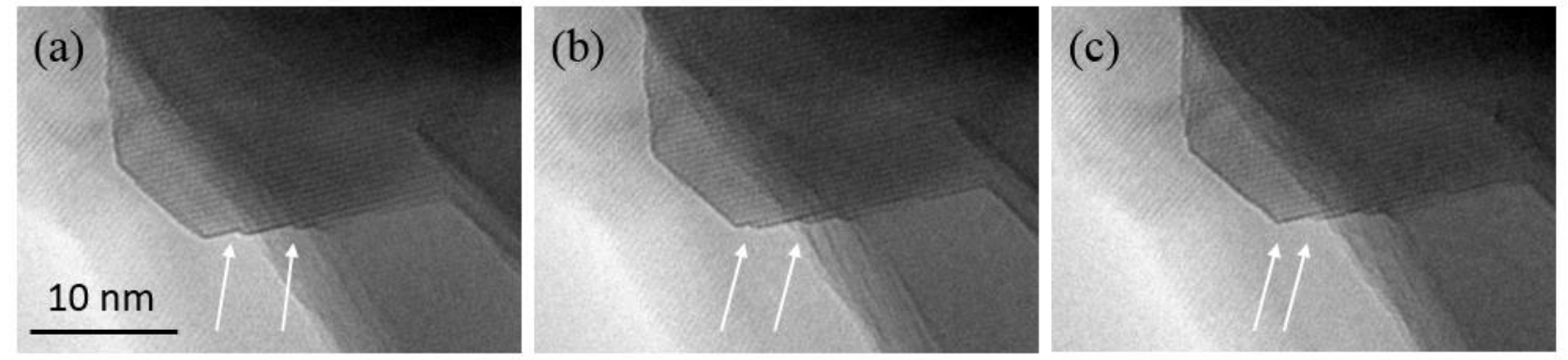

Figure 3. Oxide lattice propagation of the with time interval 2 seconds between each image. 